\title{
Anatomía foliar comparada y relaciones filogenéticas de 11 especies de Laeliinae con énfasis en Brassavola (Orchidaceae)
}

\section{Eliana Noguera-Savelli ${ }^{1} \&$ Damelis Jáuregui ${ }^{2}$}

1. Posgrado en Ciencias Biológicas, Unidad de Recursos Naturales, Centro de Investigación Científica de Yucatán, A.C. (CICY), Colonia Chuburná de Hidalgo, Calle 43. №130. Mérida, Yucatán, México C.P. 97200; eliananoguera@yahoo.com

2. Universidad Central de Venezuela, Facultad de Agronomía, Instituto de Botánica Agrícola, Laboratorio de Morfoanatomía Vegetal, Prolongación Av. 19 de abril, Maracay 2101,Estado Aragua, Venezuela; jaureguid@agr.ucv.ve

$$
\text { Recibido 05-I-2011. C Corregido 25-I-2011. Aceptado 14-II-2011. }
$$

\begin{abstract}
Comparative leaf anatomy and phylogenetic relationships of 11 species of Laeliinae with emphasis on Brassavola (Orchidaceae). Brassavola inhabits a wide altitude range and habitat types from Northern Mexico to Northern Argentina. Classification schemes in plants have normally used vegetative and floral characters, but when species are very similar, as in this genus, conflicts arise in species delimitation, and alternative methods should be applied. In this study we explored the taxonomic and phylogenetic value of the anatomical structure of leaves in Brassavola; as ingroup, seven species of Brassavola were considered, and as an outgroup Guarianthe skinneri, Laelia anceps, Rhyncholaelia digbyana and Rhyncholaelia glauca were evaluated. Leaf anatomical characters were studied in freehand cross sections of the middle portion with a light microscope. Ten vegetative anatomical characters were selected and coded for the phylogenetic analysis. Phylogenetic reconstruction was carried out under maximum parsimony using the program NONA through WinClada. Overall, Brassavola species reveal a wide variety of anatomical characters, many of them associated with xeromorphic plants: thick cuticle, hypodermis and cells of the mesophyll with spiral thickenings in the secondary wall. Moreover, mesophyll is either homogeneous or heterogeneous, often with extravascular bundles of fibers near the epidermis at both terete and flat leaves. All vascular bundles are collateral, arranged in more than one row in the mesophyll. The phylogenetic analysis did not resolve internal relationships of the genus; we obtained a polytomy, indicating that the anatomical characters by themselves have little phylogenetic value in Brassavola. We concluded that few anatomical characters are phylogenetically important; however, they would provide more support to elucidate the phylogenetic relantionships in the Orchidaceae and other plant groups if they are used in conjunction with morphological and/or molecular characters. Rev. Biol. Trop. 59 (3): $1047-$ 1059. Epub 2011 September 01.
\end{abstract}

Key words: Cladistic analysis, leaf anatomy, orchids, Brassavola, Laelia, Guarianthe, Rhyncholaelia.

Laeliinae es una subtribu neotropical que comprende alrededor de 40 géneros y 1913 especies (van den Berg 2005). Géneros como: Brassavola R. Br., Cattleya Lindl., Guarianthe Dressler \& W.E. Higgins y Rhyncholaelia Schltr., son de un extraordinario valor ornamental; otros como Encyclia Hook., Epidendrum L. y Prosthechea Knowles \& Westc., constituyen elementos florísticos comunes del Neotrópico. Brassavola (Epidendroideae, Laeliinae) se distribuye desde el norte de México hasta el norte de Argentina y ocupan intervalos de distribución altitudinal desde los 0-1000 (-1700) msnm. Entonces se caracteriza por presentar plantas epífitas con tallos teretes, de una sola hoja suculenta, e inflorescencias generalmente racemosas, de una a tres (-18) flores. Las especies de Brassavola son comúnmente conocidas como "dama de noche" por sus flores blancas, muy fragantes al anochecer. La mayoría de 
las plantas muestran un aspecto similar en sus características vegetativas y florales, lo cual ha causado conflictos en la delimitación de las especies y en los sistemas de clasificación propuestos. El número y la delimitación de las especies para el género es variable de acuerdo a diversos autores, Jones (1975), consideró 18 spp; Brieger et al. (1976), incluyeron 15 spp; por su parte Withner (1998) señaló $16 \mathrm{spp}$. y posteriormente en 2007 reconsideró el género constituido por 19 spp.

A pesar de los avances en el conocimiento de la estructura anatómica de muchos representantes de las Orchidaceae, Stern \& Carlsward (2009) señalaron que los estudios anatómicos exhaustivos en la subtribu Laeliinae han sido escasos. Sin embargo, Baker (1972) caracterizó detalladamente la morfología y anatomía foliar de 36 géneros y 79 taxones de las Laeliinae. Por otra parte, Oliveira \& Sajo (1999) estudiaron la anatomía foliar de los géneros: Prosthechea y Encyclia, además de las especies Epidendrum campestre Lindl. y E. secundum Jacq., también pertenecientes a las Laeliinae.

En Brassavola las investigaciones se han centrado principalmente en dos especies $B$. nodosa (L.) Lindl. y B. cucullata (L.) R. Br. en las cuales se ha estudiado la anatomía de sus órganos vegetativos (Abreu \& Peña 1982, Pridgeon et al. 1983, Porembski \& Barthlott 1988, Stern \& Carlsward 2009). Stern \& Carlsward (2009) con un enfoque evolutivo, realizaron un análisis cladístico basado en caracteres morfo-anatómicos vegetativos de 40 especies (incluyendo $B$. cucullata) de la subtribu Laeliinae, con el fin de examinar si la anatomía podría proporcionar evidencia sobre la monofilia del grupo. De esta manera se evaluaron seis caracteres anatómicos foliares, los resultados obtenidos indicaron que los caracteres vegetativos por si solos son insuficientes para resolver las relaciones filogenéticas entre los taxones estudiados, pero pueden ser útiles para evaluar los patrones de evolución dentro de la subtribu.

Dado que Brassavola es un grupo monofilético (van den Berg et al. 2009) y que sus plantas presentan una morfología foliar muy similar, en el presente trabajo se examinó la estructura anatómica foliar de un grupo de especies de Laeliinae con énfasis en Brassavola, con el propósito de explorar caracteres de valor taxonómico y además evaluar si éstos son filogenéticamente informativos para este género.

\section{MATERIALES Y MÉTODOS}

En clasificaciones taxonómicas previas para Brassavola (Schlechter 1919, Brieger et al. 1976, Withner 1998, 2007) la mayoría coinciden en la existencia de cuatro secciones. En este estudio se consideraron siete especies pertenecientes a tres de las secciones propuestas por estos autores: 1. Secc. Brassavola Lindl.: B. appendiculata A. Rich. \& H. G. Galeotti; 2. Secc. Cuneilabia Rchb.: B. acaulis Lindley \& Paxton, B. nodosa (L.) Lindl. y B. subulifolia Lindl.; 3. Secc. Conchoglossa Schltr.: B. ceboletta Rchb., B. fragrans Barb. Rodr. y $B$. tuberculata Hook.; basado en revisiones taxonómicas previas en este trabajo se acepta el nombre $B$. appendiculata. La especie Brassavola appendiculata se distribuye desde el Norte de México hasta Nicaragua y corresponde con lo que otros autores han llamado en esta zona como B. cucullata. Adicionalmente, se analizaron cuatro especies también pertenecientes a la subtribu Laeliinae: Guarianthe skinneri (Bateman) Dressler \& W. E. Higgins, Laelia anceps Lindl., Rhyncholaelia glauca (Lindl.) Schltr. y R. digbyana (Lindl.) Schltr. La cita de los nombres de los autores fue seguida de acuerdo a Brumitt \& Powell (1992). Los especímenes del material estudiado provienen de plantas recolectadas en el campo y de colecciones particulares. Muestras representativas de cada taxón fueron depositadas en el Herbario CICY, del Centro de Investigación Científica de Yucatán, Mérida, México, con los siguientes registros: $R$. glauca: D. Angulo sn; $R$. digbyana: G. Carnevali et al. \# 4966; $B$. subulifolia: G. Carnevali \# 7503; B. ceboletta: G. Carnevali \#7529; B. fragrans: G. Carnevali \#7530; B. nodosa: G. Carnevali \#7531; B. acaulis: G. Carnevali \#7236; G. skinneri:G. Carnevali sn; Laelia anceps: G. Carnevali sn; 
B. appendiculata: W. Cetzal \# 32; B. tuberculata: A. Pardo sn.

El estudio se llevó a cabo con hojas maduras de plantas vivas; se tomaron fragmentos de la porción media de la hoja y se fijaron en FAA (Formaldehido 5\%, Ácido acético 5\%, Agua $40 \%$ y Etanol 50\%) (Sass 1958). Dichos fragmentos fueron seccionados transversalmente a mano alzada. Las secciones fueron teñidas con azul de tolouidina acuosa $(0.5 \%)$, se montaron en glicerina $(50 \%)$ y se sellaron con esmalte transparente para uñas. Para el estudio epidérmico, se colocaron porciones de hoja de aproximadamente $0.5 \mathrm{~cm}$ en hipoclorito de sodio comercial $(3.75 \%)$ durante tres horas, se lavaron exhaustivamente con agua destilada y se eliminaron los restos del mesofilo con ayuda de un pincel y bajo un microscopio estereoscópico. Las porciones de epidermis se tiñeron y se montaron de forma semejante a lo antes expuesto. Todas las láminas preparadas fueron fotografiadas con un microscopio óptico Nikon Eclipse E 200 equipado con cámara fotográfica digital Evolution LC en el Laboratorio de Anatomía Vegetal "Antonio Fernández", de la Facultad de Agronomía de la Universidad Central de Venezuela (UCV), en Maracay, Venezuela. Por otra parte, se observaron con un microscopio óptico binocular Leica-DME para la descripción de los caracteres de los tejidos dérmico, fundamental y vascular, en el Laboratorio de Anatomía Vegetal de la Unidad de Recursos Naturales del Centro de Investigación Científica de Yucatán, A.C. (CICY), Mérida, Yucatán, México.

Producto de la caracterización y observación de la estructura anatómica foliar se codificaron 10 caracteres, los mismos definieron sus estados en cada caso (Cuadro 1). Una vez codificados todos los caracteres para cada taxa, se procedió a construir la matriz que se emplearía en el análisis cladístico (Cuadro 2). Los caracteres codificados fueron no ordenados, discretos, binarios y multiestados. Las especies: G. skinneri, L. anceps, R. glauca y $R$. digbyana se seleccionaron como grupo externo, esto basado en la filogenia de las Laeliinae realizada por van den Berg et al. (2009); para la polarización de los caracteres se utilizó como raíz a $L$. anceps. La reconstrucción filogenética se efectuó con el uso de NONA (Goloboff 1993) a través de WinClada (Nixon 2002). Por lo tanto, se aplicó el algoritmo Ratchet (Nixon 1999) con 500 iteraciones, diez árboles y tres caracteres muestreados en cada iteración. Cuando se obtuvo más de un árbol igualmente parsimonioso, se calcularon árboles de consenso estricto para identificar los clados comunes a todos estos árboles. La discriminación entre los árboles resultantes se hizo sobre las bases

CUADRO 1

Caracteres anatómicos y estado de carácter codificados para el análisis cladístico de Brassavola

TABLE 1

Anatomical characters and character state coded for cladistic analysis of Brassavola

$\begin{array}{cl}\mathrm{N}^{\circ} & \text { Caracter anatómico/estado de caracter } \\ 1 & \text { Superficie de la cutícula: lisa (0), ondulada (1) } \\ 2 & \text { Distribución de los estomas: abaxial (0), ad-abaxial (1) } \\ 3 & \text { Tipo de tricomas: tetracítico (0), paracítico (1), anomocíticos (2) } \\ 4 & \text { Células de pared secundaria con engrosamientos: ausentes (0), presentes (1) } \\ 5 & \text { Disposición de los paquetes de fibras: adaxial/abaxial (0), abaxial (1) } \\ 6 & \text { Presencia de rafidios: ausentes (0), presentes (1) } \\ 7 & \text { Presencia de drusas: ausentes (0), presentes (1) } \\ 8 & \text { Distribución de hipodermis foliar: adaxial (0), adaxial-abaxial (1), ausente (2) } \\ 9 & \text { Organización del mesófilo: heterogéneo (0) homogéneo (1) } \\ 10 & \text { Tipo de mesófilo: equifacial (0), bifacial (1) }\end{array}$


CUADRO 2

Estados de caracter codificados para cada taxa en el análisis cladístico de Brassavola y su grupo externo

TABLE 2

Coded character states for each taxa in the cladistic analysis of Brassavola and the outgroup

\begin{tabular}{lcccccccccc}
\multicolumn{1}{c}{ Taxon } & \multicolumn{11}{c}{ Caracteres } \\
& 1 & 2 & 3 & 4 & 5 & 6 & 7 & 8 & 9 & 10 \\
Laelia anceps & 0 & 0 & 0 & 0 & 0 & 0 & 0 & 0 & 0 & 0 \\
Gurianthe skinneri & 0 & 0 & 1.2 & 0 & 1 & 0 & 1 & 0 & 0 & 1 \\
Rhyncholaelia digbyana & 0 & 0 & 0 & 1 & 1 & 0 & 1 & 2 & 0 & 1 \\
R. glauca & 0 & 0 & 1 & 0 & 1 & 1 & 1 & 2 & 0 & 1 \\
Brassavola acaulis & 1 & 1 & 1 & 1 & 1 & 1 & 0 & 2 & 1 & 0 \\
B. appendiculata & 0 & 1 & 1 & 0 & 1 & 1 & 0 & 1 & 0.10 .2 \\
B. ceboletta & 1 & 1 & 1 & 1 & 0 & 0 & 0 & 2 & 0 & 0 \\
B. fragrans & 0 & 1 & 0,1 & 1 & 0 & 0 & 0 & 1 & 1 & 0 \\
B. nodosa & 1 & 1 & 0,1 & 1 & 1 & 1 & 0 & 1 & 0 & 0 \\
B. tuberculata & 0 & 1 & 1 & 0 & 0 & 1 & 0 & 2 & 1 & 0 \\
B. subulifolia & 0 & 1 & 1 & 0 & 0 & 1 & 0 & 2 & 0 & 0
\end{tabular}

de longitud de los árboles (L), el índice de consistencia (IC) (Kluge \& Farris 1969) y el índice de retención (IR) los cuales son valores que evalúan la homoplasia del juego de datos (Farris 1989).

\section{RESULTADOS}

Estudio anatómico foliar: Sistema dérmico (Cuadro 3): Cutícula: tanto las especies de Brassavola como las del grupo externo tienen cutícula gruesa, sus espesores superan los $10 \mu \mathrm{m}$ (evaluación efectuada sobre fotografías), la cual varía de lisa a ondulada (Figs. 1-2). CÉlULAS EPIDÉRMICAS TÍPICAS: isodiamétricas a ligeramente alargadas en vista paradérmica con paredes anticlinales rectas y gruesas, mientras que en sección transversal se observan cuadrangulares a rectangulares con la pared periclinal externa plana o convexa en mayor o menor grado y llegan a formar papilas en Brassavola acaulis, Brassavola ceboletta (Fig. 3) y Brassavola nodosa (Fig. 4). TRicomas: ausentes en todas las especies estudiadas. Estomas: las hojas de Brassavola son anfiestomáticas, sin embargo el resto de las especies estudiadas tienen hojas hipoestomáticas (Cuadro 3). El tipo de estoma en Brassavola es variable: paracíticos, tetracíticos o combinaciones de éstos, los cuales pueden estar al mismo nivel del resto de las células epidérmicas o hundidos. En B. acaulis (Fig. 5), B. appendiculata, B. tuberculata, B. subulifolia (Fig. 6) y B. ceboletta, son paracíticos; en Brassavola fragrans y $B$. nodosa son paracíticos y tetracíticos; en

CUADRO 3

Carácteres del sistema dérmico de las especies de Brassavola y su grupo externo

TABLE 3

Characters of Brassavola dermal system and the outgroup

\begin{tabular}{lcccc}
\multicolumn{1}{c}{ Taxon } & $\begin{array}{c}\text { Forma de } \\
\text { la hoja }\end{array}$ & $\begin{array}{c}\text { Superficie de } \\
\text { la cutícula }\end{array}$ & Disposición de los estomas & Tipo de estomas \\
L. anceps & plana & lisa & abaxial & tetracíticos \\
G. skinneri & plana & lisa & abaxial & anomocíticos/paracíticos \\
R. digbyana & plana & lisa & abaxial & tetracíticos \\
R. glauca & plana & lisa & abaxial & paracíticos \\
B. acaulis & terete & ondulada & adaxial-abaxial & paracíticos \\
B. appendiculata & terete & lisa & adaxail-abaxial & paracíticos \\
B. ceboletta & plana & ondulada & adaxial-abaxial & paracíticos \\
B. fragrans & terete & lisa & adaxial-abaxial & paracíticos/tetracíticos \\
B. nodosa & plana & ondulada & adaxial-abaxial & paracíticos/tetracíticos \\
B. tuberculata & terete & lisa & adaxial-abaxial & paracíticos
\end{tabular}



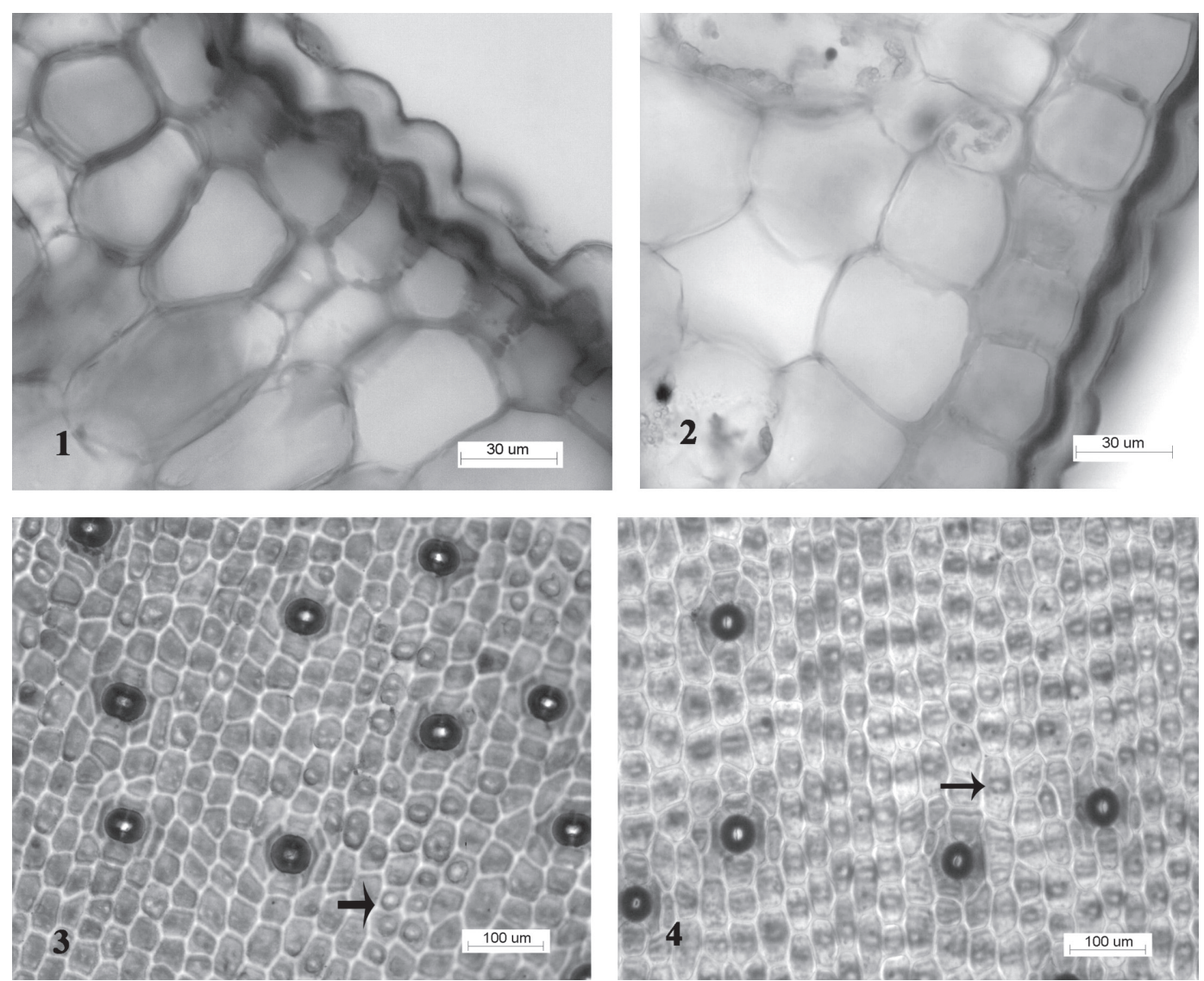

Fig. 1-4. Secciones transversales de la hoja de Brassavola. Nótese pared periclinal externa de células epidérmicas y cutícula. 1. B. acaulis. convexa y muy ondulada 2. B. fragrans. plana y levemente ondulada. 3. Vistas paradérmicas. Nótense papilas, indicadas con flecha en 3. B. ceboletta y 4. B. nodosa.

Fig. 1-4. Transverse sections of Brassavola leaf. Note outer periclinal wall of epidermal cells and cuticule. 1. B. acaulis. Convex an very undulating. 2. B. fragrans. flat and slightly undulating. Paradermal views. Note papillae, as indicated by arrow in 3. B. ceboletta and 4. B. nodosa.

Rhyncholaelia digbyana y Laelia anceps son tetracíticos y en Rhyncholaelia glauca (Fig. 7) son paracíticos (Cuadro 3). Sólo en Guarianthe skinneri se diferencian estomas anomocíticos (Fig. 8) además de los paracíticos. Sistema Fundamental: (Cuadro 4). Hipodermis: en las especies estudiadas que tienen hipodermis, esta es uniestratificada a excepción de G. skinneri que la presenta biestratificada, pero sólo en la cara adaxial. En las hojas teretes de B. appendiculata (Fig. 9) y B. fragrans, la hipodermis se distribuye en la cara abaxial y adaxial, por consiguiente circunda la hoja, mientras que las hojas conduplicadas de B. nodosa, se ubica en ambas caras. En L. anceps, se dispone sólo en la cara adaxial (Fig. 10). Las células de este tejido son isodiamétricas o en ocasiones ligeramente alargadas periclinal o anticlinalmente y con paredes engrosadas. ClorénQuima: a pesar que algunas hojas de Brassavola son teretes o semiteretes, se puede diferenciar un lado adaxial correspondiente al área superficial sulcada de la hoja y uno abaxial que corresponde a la región convexa o redondeada de la misma hoja. Por lo tanto se observaron dos tipos de hoja de acuerdo a la disposición del parénquima: 

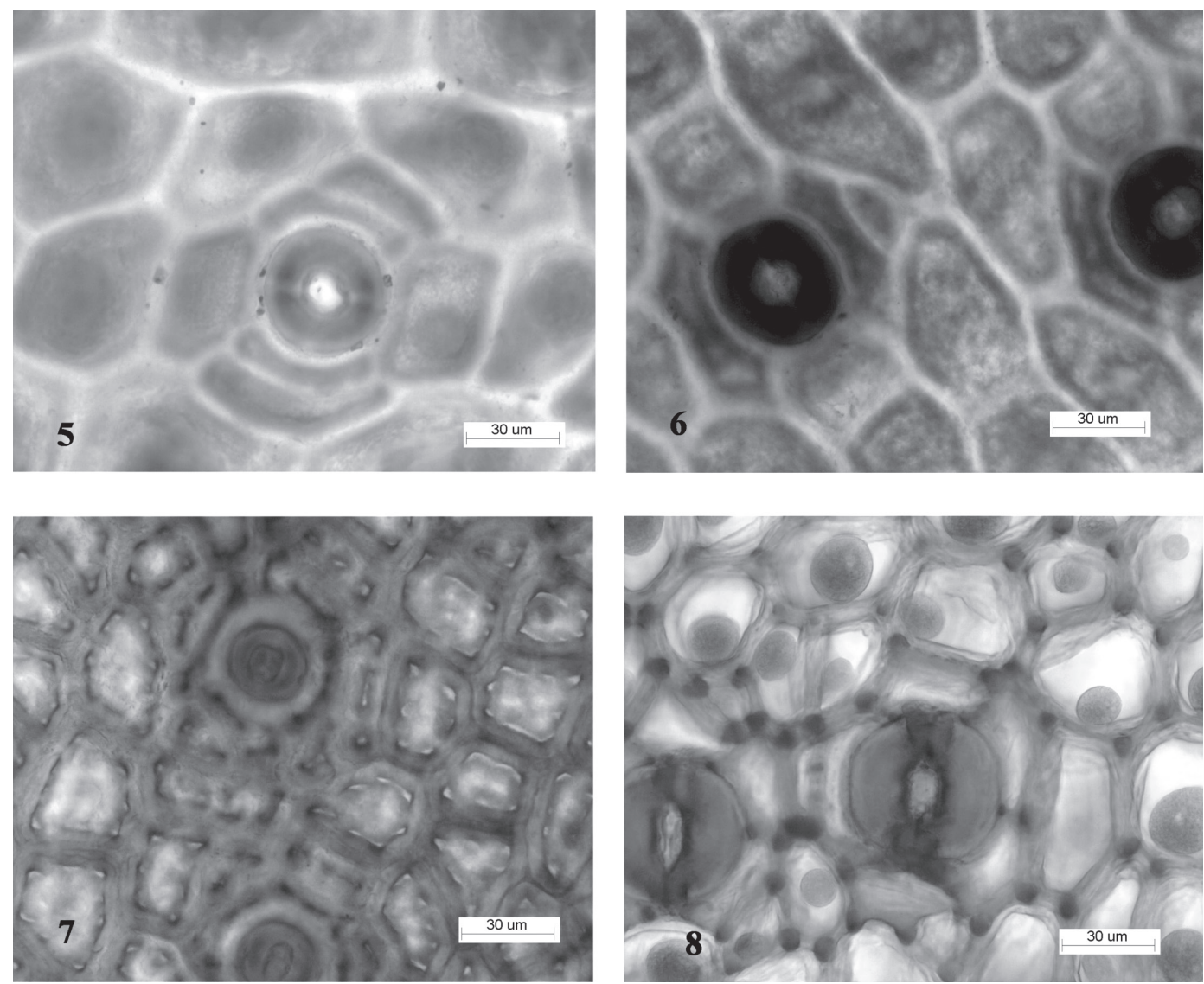

Fig. 5-8. Vistas paradérmicas de la epidermis foliar. 5. Brassavola acaulis. 6. B. subulifolia. 7. Ryncholaelia glauca. 8. Guarianthe skinneri.

Fig. 5-8. Paradermal views of leaf epidermis. 5. Brassavola acaulis. 6. B. subulifolia. 7. Ryncholaelia glauca. 8. Guarianthe skinneri.

bifaciales y equifaciales. En G. skinneri, $R$. digbyana y $R$. glauca, el mesofilo es bifacial, con una clara diferenciación de parénquima en empalizada, conformado por tres a seis capas de células alargadas y de parénquima esponjoso constituido por nueve a trece capas de células, voluminosas y de paredes delgadas (Cuadro 4). El resto de las especies poseen hojas equifaciales, unas con diferenciación de parénquima empalizada y parénquima esponjoso en ambas caras: B. ceboletta, B. nodosa y L. anceps; y se presentaron generalmente cinco a seis capas del primer tejido en la cara adaxial y cuatro a cinco en la abaxial con siete a diez capas del esponjoso en el centro, este último constituido por células voluminosas y de paredes delgadas, y las otras: B. acaulis, B. fragrans, B. tuberculata y $B$. subulifolia con mesofilo homogéneo ( $\sin$ distinción entre parénquima empalizada y esponjoso) (Cuadro 4); estas últimas especies tienen hojas de semiteretes a teretes y el mesofilo está integrado por aproximadamente 20-22 capas de células. En B. appendiculata el mesofilo puede ser heterogéneo u homogéneo. (Cuadro 4). CÉlulas CON ENGROSAMIENTOS: espiralados en la pared secundaria de las células del mesofilo son muy abundantes en: B. acaulis (Fig. 11), B. ceboletta, B. fragrans 
CUADRO 4

Carácteres del sistema fundamental y vascular de las especies de Brassavola y su grupo externo

TABLE 4

Fundamental characters and vascular system of Brassavola species and the outgroup

\begin{tabular}{|c|c|c|c|c|c|c|c|c|c|}
\hline \multirow[b]{2}{*}{ Taxon } & \multicolumn{2}{|c|}{ Hipodermis } & \multirow{2}{*}{$\begin{array}{l}\text { Tipo de hoja de } \\
\text { acuerdo al mesofilo }\end{array}$} & \multirow[b]{2}{*}{ Mesófilo } & \multirow{2}{*}{$\begin{array}{l}\text { Células con } \\
\text { engrosamientos } \\
\text { espiralados }\end{array}$} & \multicolumn{2}{|c|}{ Cristales } & \multicolumn{2}{|c|}{ Fibras extravasculares } \\
\hline & adaxial & adaxial-abaxial & & & & rafidios & drusas & abaxial & adaxial-abaxial \\
\hline L. anceps & $\mathrm{P}\left(1^{*}\right)$ & & equifacial & $\mathrm{He}$ & A & $\mathrm{P}$ & A & & $\mathrm{P}$ \\
\hline G. skinneri & $\mathrm{P}\left(2^{*}\right)$ & & bifacial & $\mathrm{He}$ & A & $P$ & $\mathrm{P}$ & $\mathrm{P}$ & \\
\hline R. digbyana & & A & bifacial & $\mathrm{He}$ & $P$ & $P$ & $\mathrm{P}$ & $P$ & \\
\hline R. glauca & & $\mathrm{A}$ & bifacial & $\mathrm{He}$ & A & A & $\mathrm{P}$ & $\mathrm{P}$ & \\
\hline B. acaulis & & A & equifacial & Ho & $\mathrm{P}$ & A & A & $\mathrm{P}$ & \\
\hline B. appendiculata & & $\mathrm{P}\left(1^{*}\right)$ & equifacial/bifacial & $\mathrm{Ho} / \mathrm{He}$ & A & A & A & $\mathrm{P}$ & \\
\hline B. ceboletta & & A & equifacial & $\mathrm{He}$ & $P$ & $P$ & A & & $\mathrm{P}$ \\
\hline B. fragrans & & $\mathrm{P}\left(1^{*}\right)$ & equifacial & Ho & $\mathrm{P}$ & $\mathrm{P}$ & A & & $\mathrm{P}$ \\
\hline B. nodosa & & $\mathrm{P}\left(1^{*}\right)$ & equifacial & $\mathrm{He}$ & $P$ & A & $\mathrm{A}$ & $\mathrm{P}$ & \\
\hline B. tuberculata & & A & equifacial & Но & A & A & A & & $\mathrm{P}$ \\
\hline B. subulifolia & & A & equifacial & Ho & A & A & A & & $\mathrm{P}$ \\
\hline
\end{tabular}

He=heterogéneo; $\mathrm{Ho}=$ homogeneo; $\mathrm{P}=$ presente $;{ }^{*}=$ número de capas; $\mathrm{A}=$ ausente.

y B. nodosa; mientras que en $R$. digbyana, son reticulados y muy engrosados. En el resto de las especies estudiadas no se observaron dichos engrosamientos (Cuadro 4). INCLUSIONES CELULARES: idioblastos conteniendo cristales de oxalato de calcio en forma de rafidios en el mesofilo de: B. ceboletta (Fig. 12), B. fragrans, L. anceps, G. skinneri y $R$. digbyana; o con drusas en la hipodermis y el mesofilo de: $G$. skinneri, $R$. digbyana y $R$. glauca (Cuadro 4). FIBRAS EXTRAVASCULARES: son comunes en todas las especies estudiadas, los paquetes de fibras son variables en posición y número. Dispuestos en la cara abaxial: B. acaulis, B. appendiculata, $B$. nodosa, G. skinneri, $R$. digbyana y $R$. glauca y organizados en ambas caras en: B. ceboletta, B. fragrans, B. tuberculata, B. subulifolia y L. anceps (Cuadro 4) (Fig. 13). En las hojas teretes a semiteretes se observó una mayor cantidad de paquetes de fibras extravasculares. SisTEMA VASCULAR: HAZ VASCULAR: de tipo colateral cerrado. En Brassavola los haces principales generalmente se disponen en un arco central y los haces vasculares secundarios, en $\operatorname{arcos}$ subyacentes superior y/o inferior, arreglados en zig-zag. En L. anceps, G. skinneri, R. digbyana y $R$. glauca, los haces vasculares principales se organizan en una hilera central, intercalados con haces vasculares secundarios. Estos haces están bordeados por una vaina vascular esclerenquimática, más desarrollada hacia el lado del floema (Fig. 13).

Análisis filogenético de Brassavola: La matriz de datos anatómicos comprendió diez caracteres (Cuadro 2). En la búsqueda heurística se encontraron 168 árboles más parsimoniosos de 22 pasos, un $\mathrm{IC}=0.50$ y un $\mathrm{IR}=0.57$. En el árbol de consenso estricto no se resuelven las relaciones internas del género, se obtuvo una politomía (Fig. 14).

\section{DISCUSIÓN}

Los caracteres presentes en la cutícula de las especies de Brassavola coinciden con las características señaladas para las Laeliinae por Baker (1972) al referirse a la presencia 

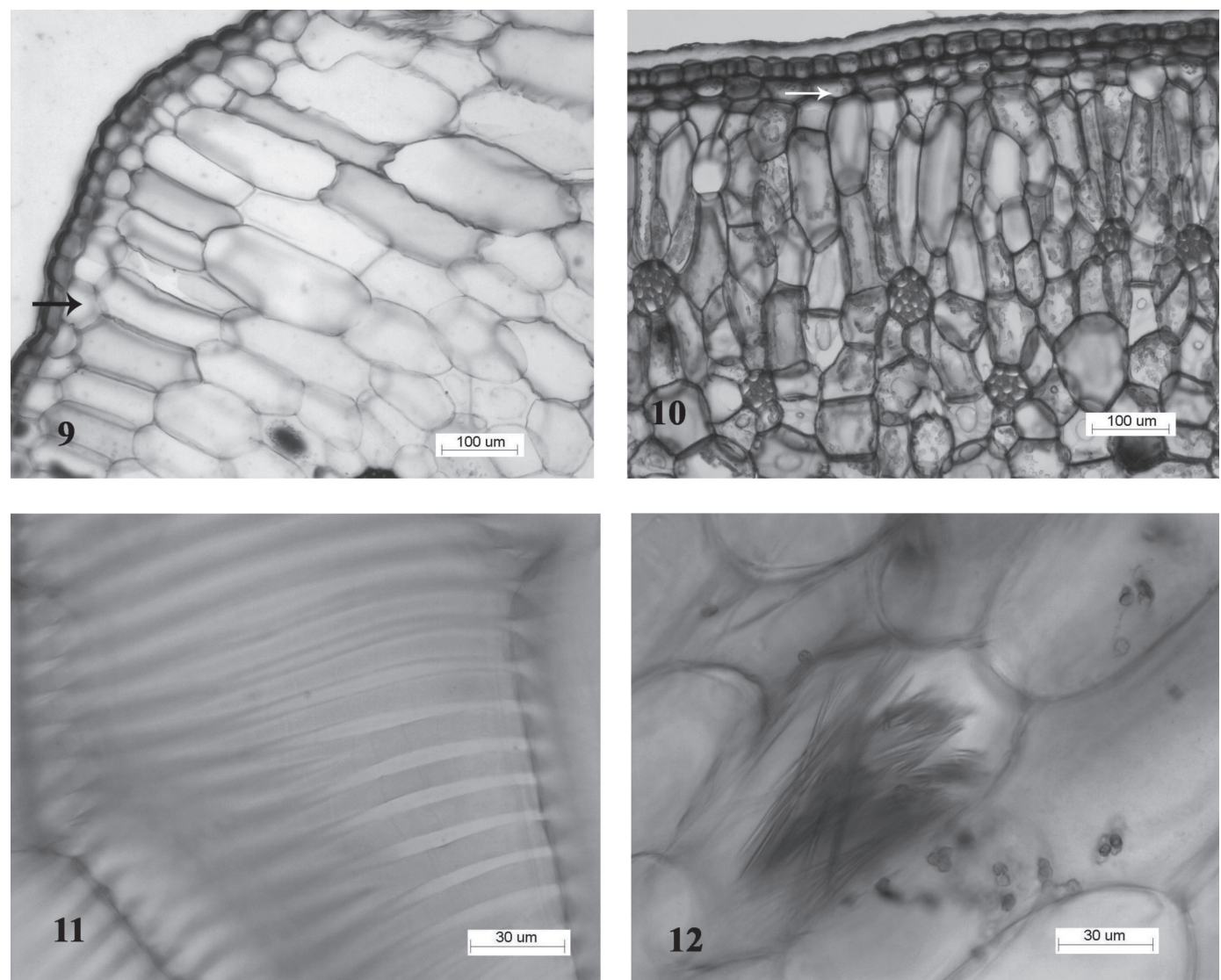

Fig. 9-12. Secciones transversales de las hojas. 9. Brassavola appendiculata, nótese hipodermis (indicada con flecha) y parénquima clorofiliano. 10. Laelia anceps con mesofilo equifacial heterogéneo. 11. B. acaulis, con engrosamientos espiralados. 12. B. ceboletta, nótense inclusiones cristalinas.

Fig. 9-12. Transverse sections of leaves. Brassavola appendiculata. note hypodermis (indicated by arrow) and chorophyll parenchyma. 10. Laelia anceps. with mesophyll equifacial heterogeneous. 11. B. acaulis. note spiral thickenings. 12 . $B$. ceboletta. note crystalline inclusions.

de una cutícula de lisa a granular. En este estudio se caracterizó como lisa u ondulada hasta papilosa. Sandoval-Zapotitla et al. (2003) mencionaron que la presencia de micropapilas como ornamentaciones epidérmicas facilita una mejor captación de luz por diferentes ángulos oblicuos. Asimismo, pueden actuar en la regulación de la cantidad de luz incidente y evitan que se calienten los tejidos subyacentes y se dañen los estomas (Lindorf et al. 1991). En Brassavola, la presencia de papilas estuvo asociada a las especies con hojas planas y erectas de $B$. ceboletta y semiteretes de $B$. nodosa, sin embargo, también se presentaron en $B$. acaulis, que tienen hojas teretes. De acuerdo a Esau (1965) y Withner et al. (1974) el grosor de la cutícula está determinado por la exposición al sol, las hojas más expuestas tienden a exhibir una cutícula más gruesa en ambas caras; este rasgo ha sido observado en otras orquídeas, como Maxillaria miniata y Pleurothalis cardiantha (Ely et al. 2007). Esta condición se constató en Brassavola, debido a que crecen generalmente en las ramas más 


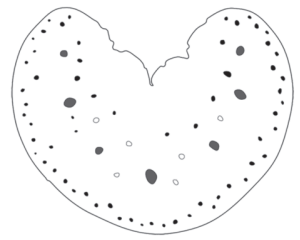

A

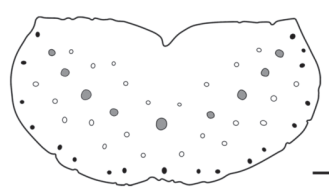

$\mathrm{E}$

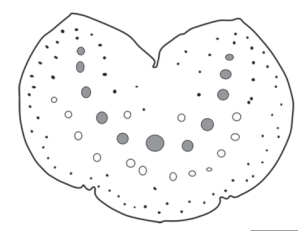

B

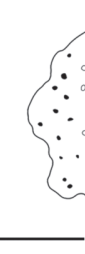

$3 \mathrm{~mm}$

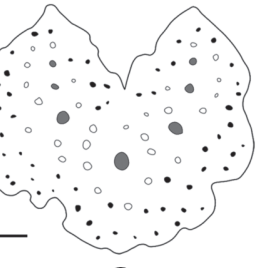

C

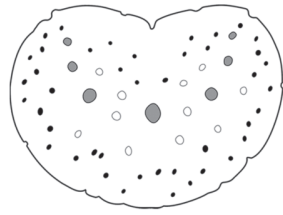

D

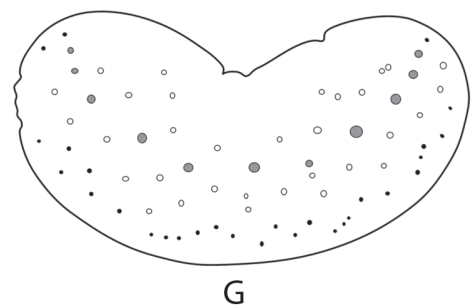

$\mathrm{F}$

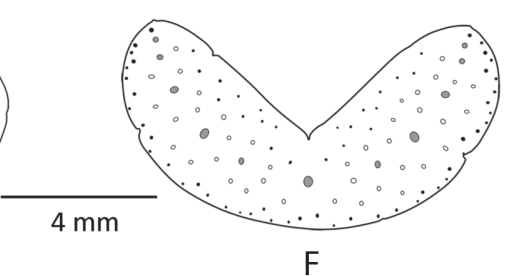

Fig. 13. Comparación de secciones transversales de las especies de Brassavola. A-D. aplica la escala: $3 \mathrm{~mm}$. A. $B$. appendiculata. B. B. fragrans. C. B. revoluta. D. B. subulifolia. E-G. aplica la escala: $4 \mathrm{~mm}$. E. B. acaulis. F. B. ceboletta. G. B. nodosa.

Fig. 13. Comparison of transverse sections of species of Brassavola. A-D. scale applies: $3 \mathrm{~mm}$. A. B. appendiculata. B. $B$. fragrans. C. B. revoluta. D. B. subulifolia. E-G. scale applies: $4 \mathrm{~mm}$. E. B. acaulis. F. B. ceboletta. G. B. nodosa.

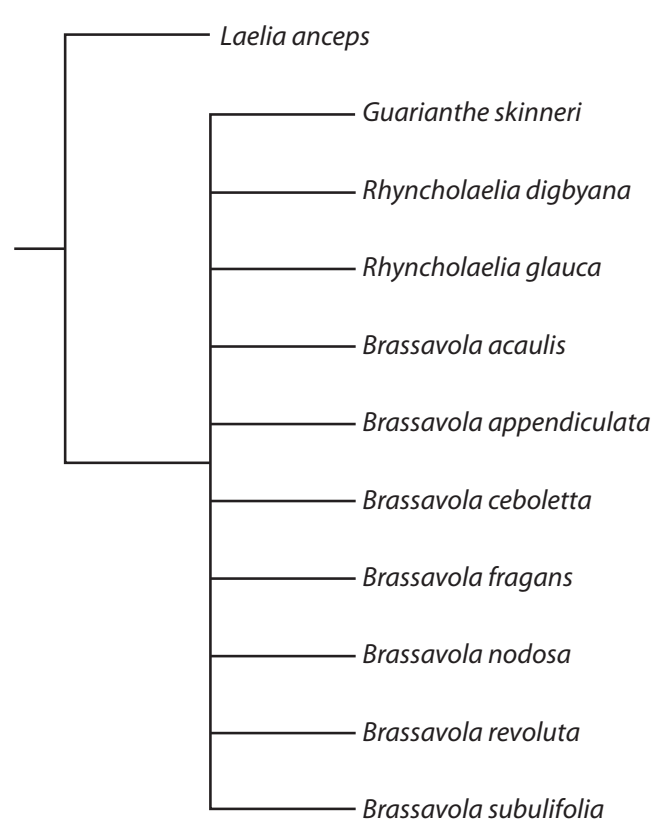

Fig. 14. Árbol de consenso estricto de 168 árboles más parsimoniosos basado en caracteres anatómicos foliares de siete especies de Brassavola. $\mathrm{L}=22, \mathrm{CI}=50, \mathrm{RI}=57$.

Fig. 14. Strict consensus tree of 168 most parsimonious trees base on leaf anatomical characters of seven species of Brassavola. $\mathrm{L}=22, \mathrm{IC}=50, \mathrm{IR}=57$. expuestas del forofito y presentan cutículas gruesas, superiores a $7 \mu \mathrm{m}$ (considerando la propuesta de Pyykkö 1966), además tienen las paredes anticlinales y periclinales de las células epidérmicas engrosadas. No se observaron tricomas en Brassavola y el resto de las especies estudiadas, esto en correspondencia con los resultados de Stern \& Carlsward (2009), quienes mencionaron la ausencia de tricomas en las Laeliinae. Sin embargo, Baker (1972) indicó que es frecuente la presencia de tricomas en las Laeliinae, por lo que Stern \& Carlsward (2009) argumentaron que la ausencia de tricomas en las 37 especies estudiadas, posiblemente se debía a que la célula terminal de dichos tricomas tiene paredes delgadas y se desprende fácilmente, además mencionaron que estos tricomas son efímeros en las hojas adultas. No obstante, lo anteriormente expuesto es poco probable porque deberían observarse las bases de los mismos en la epidermis. Los estomas paracíticos y tetracíticos fueron los más frecuentes, lo cual coincide con lo descrito por Stern \& Carlsward (2009), quienes señalaron que los estomas tetracíticos son los 
más comunes en las Laeliinae. En el trabajo de los autores antes citados no se describieron los tipos de estomas de cada una de las especies consideradas, de igual forma ocurre con el estudio de Abreu \& Peña (1982). Por consiguiente, en esta investigación se presenta una primera descripción de los tipos de estomas para siete especies de Brassavola y para las especies $G$. skinneri, L. anceps, $R$. glauca y $R$. digbyana.

En algunas especies de orquídeas que carecen de seudobulbos, el mesofilo foliar posee células con engrosamientos espiralados, que tienen por función almacenar agua y evitar el colapso de los tejidos durante los períodos de sequía, tal como se presenta en la subtribu Pleurothallidinae (Pridgeon 1986, Scatena \& Nunes 1996, Ely et al. 2007). Este rasgo se observó en B. acaulis y B. ceboletta. En Brassavola los tallos son muy delgados, teretes y posiblemente sea muy limitada su función de almacenamiento de agua, por consiguiente, la hoja está adaptada para cumplir esta función. Madisson (1977) y Roth (1992) consideraron que la hipodermis es la estructura más común para el almacenamiento foliar de agua, estando presente en varias familias de epífitas. Según Haberlandt (1914), Madisson (1977) y Ely et al. (2007), además de almacenar agua, la hipodermis ejerce un papel importante en la economía de calor especialmente en epífitas con metabolismo CAM y protege al tejido fotosintético del exceso de luz, al disminuir la cantidad de ésta que incide sobre el clorénquima.

La constitución del mesofilo está asociada a la forma de la hoja; por lo general las hojas teretes presentan mesofilo homogéneo, no presentando diferenciación entre parénquima en empalizada y esponjoso (Oliveira \& Sajo 1999, Stern \& Carlsward 2009) y las hojas conduplicadas pueden presentar mesofilo heterogéneo, donde se distingue parénquima en empalizada y esponjoso. La mayoría de las especies estudiadas de Brassavola que presentaron parénquima homogéneo, poseen hojas teretes o semiteretes. La deposición de inclusiones celulares cristalinas ha sido frecuentemente observada en Orchidaceae (Sandoval-Zapotitla et al. 2003, Stern et al. 2004, Stern \& Carslward
2009). No obstante, poco se conoce sobre la función(es) de los cristales. Stern \& Carlsward (2009) notaron la presencia de paquetes de fibras en muchos taxones de Laeliinae. La distribución de las fibras observadas en este estudio, corresponde con la señalada por Stern \& Carlsward (2009) para L. anceps, R. digbyana, G. skinneri y B. cucullata (B. appendiculata en este estudio). Oliveira \& Sajo (1999) indicaron que las células esclerificadas confieren resistencia mecánica en caso de deshidratación, por otra parte Withner et al. (1974), señalaron que en Orchidaceae la presencia de hojas coriáceas, son características de formas vegetativas más evolucionadas, que poseen seudobulbos y son capaces de sobrevivir en hábitats xéricos. Conforme con los caracteres señalados por Rudall (1992), los resultados observados en las especies estudiadas indican que estas plantas poseen rasgos xeromórficos, puesto que la mayoría de la especies tienen cutícula gruesa, hipodermis, células con engrosamientos espiralados en el mesofilo y abundantes paquetes de fibras. Los caracteres anatómicos foliares evaluados fueron muy heterogéneos dentro del género y a su vez compartidos con otros taxones de las Laeliinae previamente estudiados a nivel específico, por consiguiente, no aportaron información de valor taxonómico para Brassavola. En el caso de las Laeliinae y específicamente en Brassavola, la variabilidad anatómica observada en sus representantes está posiblemente asociada al amplio rango de hábitats donde crecen.

Stern \& Carlsward (2009) realizaron un análisis filogenético con caracteres anatómicos vegetativos a nivel genérico en las Laeliinae, sin embargo, no obtuvieron resolución en las relaciones, concluyendo que dichos caracteres no fueron filogenéticamente informativos. Los resultados del análisis filogenético realizado en este trabajo tampoco presentaron resolución a nivel inter e intragenérico, por lo que conllevan a la siguiente interrogante $i$ En el resto de los géneros de las Laeliinae los caracteres anatómicos permiten resolver las relaciones filogenéticas intragenéricas? Es importante realizar estudios donde se explore la utilidad de los 
caracteres anatómicos para facilitar la interpretación de la evolución de estos caracteres y el entendimiento de las relaciones entre taxones.

Los caracteres anatómicos evaluados presentaron poco valor filogenéticamente informativo en Brassavola. Por otra parte, análisis filogenéticos en diferentes grupos de las Orchidaceae, como Oncidiinae (Stern \& Carlsward 2006) y Maxillarieae (Stern et al. 2004); ambos basados en ocho a diez caracteres anatómicos de la hoja, tallo y/o raíz, generaron poca resolución en las topologías. Estas investigaciones coinciden en señalar que los caracteres anatómicos generalmente son pocos y que por sí solos presentan limitada información filogenética. Sin embargo, es importante destacar que a excepción del trabajo aquí presentado, en la mayoría de los estudios arriba citados el número de taxa excede ampliamente el número de caracteres, lo cual dificulta la obtención de la resolución y soporte en las topologías. En conclusión, los caracteres anatómicos de las siete especies de Brassavola resultaron ser homogéneos y no aportaron suficiente información que permitiera separar las especies y además, estos mismos caracteres no fueron filogenéticamente informativos. No obstante, en conjunción con caracteres morfológicos y/o caracteres moleculares, pueden permitir dilucidar con mayor soporte las relaciones filogenéticas en las Orchidaceae y de otros grupos de plantas. Así por ejemplo, Cameron et al. (1999) sugirieron que las tribus Cymbidieae y Maxillarieae están cercanamente relacionadas de acuerdo a datos de secuencias de $\operatorname{rbc} L$, y posteriormente Yukawa \& Stern (2002) consideraron que los estégmatas presentes en la raíz de especies de estas subtribus podría ser una sinapomorfía en el clado Cymbidieae-Maxillarieae. Ahora bien, Prychid et al. (2004), señalaron que los cuerpos de sílice están ausentes en dos subfamilias de Orchidaceae: Vanilloideae y Orchidoideae, y en la mayoría de las tribus y subtribus de las Epidendroideae, pero están presentes en las Apostasioideae que representa el grupo hermano del resto de las Orchidaceae. Estos resultados anteriormente expuestos conducen a la interpretación que los caracteres anatómicos en general pueden ser de utilidad taxonómica y/o filogenética a diferentes niveles y dependiendo del grupo en estudio.

\section{AGRADECIMIENTOS}

A la Secretaría de Relaciones Exteriores de México, por la beca N NOGELI73101008 otorgada a la primera autora para realizar estudios de doctorado. A Diego Angulo, Germán Carnevali, William Cetzal y Alexis Pardo por el material vegetativo facilitado para la realización del estudio. Al Personal del Laboratorio de Anatomía Vegetal "Antonio Fernández" de la Facultad de Agronomía de la Universidad Central de Venezuela. A Ivón Ramírez y Rodrigo Duno por sus comentarios al documento original. A William Cetzal, Lizandro Peraza y José Luis Tapia por la revisión y sus comentarios de este manuscrito. A Silvia Hernández por el procesamiento del material depositado en el Herbario CICY. A William Cetzal por la elaboración de la ilustración.

\section{RESUMEN}

Brassavola crece en un amplio rango altitudinal y de tipos de hábitat desde el Norte de México hasta el Norte de Argentina. En los sistemas de clasificación de las plantas se utilizan normalmente caracteres vegetativos y florales, pero cuando las especies son muy similares, como es el caso de este género, los conflictos surgen en la delimitación de las especies, por lo tanto deben ser aplicados métodos alternativos de identificación. En este trabajo se exploró el valor taxonómico y filogenético de la estructura anatómica de las hojas de Brassavola, se consideró como grupo interno a siete especies de este género y como grupo externo a Guarianthe skinneri, Laelia anceps, Rhyncholaelia digbyana y Rhyncholaelia glauca. Entonces se realizaron secciones transversales de hojas frescas para el estudio de los caracteres anatómicos. Diez caracteres anatómicos vegetativos fueron seleccionados y codificados para el análisis filogenético. La reconstrucción filogenética se llevó a cabo bajo el principio de máxima parsimonia utilizando el programa NONA a través de WinClada. Todas las especies son anatómicamente similares, no obstante, difieren en algunos rasgos como presencia o no: de papilas epidérmicas, de hipodermis, de células con engrosamientos espiralados en la pared secundaria de las células del mesofilo, de inclusiones cristalinas; además en el tipo de hoja de acuerdo al arreglo del mesofilo; en la organización de los haces vasculares y de los paquetes de fibras extravasculares. 
En el árbol de consenso estricto se obtuvo una politomía. Asimismo, fue evidente que los caracteres anatómicos analizados son filogenéticamente poco informativos; sin embargo, en conjunción con caracteres morfológicos y/o moleculares, podrían dilucidar las relaciones filogenéticas.

Palabras clave: anatomía foliar, análisis cladístico, orquídeas, Brassavola, Laelia, Guarianthe, Rhyncholaelia

\section{REFERENCIAS}

Abreu, C. \& C. Peña. 1982. Estudio anatómico de hoja y tallo de orquídeas que habitan en ambiente de bosque seco. Trabajo especial de grado, Universidad Católica Andrés Bello, Caracas D.F., Venezuela.

Baker, R. 1972. Foliar anatomy of the Laeliinae (Orchidaceae). Tesis de Doctorado, Universidad de Washington, Washington, EEUU.

Cameron, K.M., W.M. Whitten, P.J. Kores, D.C. Jarell, V.A. Albert, T. Yukawa, H.G. Hills \& D.H. Goldman. 1999. A phylogenetic analysis of the Orchidaceae. Evidence from rbcL nucleotide sequences. Am. J. Bot. 86: 208-224.

Brieger, F., R. Maatsch \& K. Senghas. 1976. Die Orchideen, Lieferrung 8-12 (Schlechter III in text). Paul Parey, Berlin y Hamburgo, Alemania.

Brumitt, R. \& C. Powell. 1992. Authors of plant names. Kew, Royal Botanical Gardens, Londres, Reino Unido.

Ely, F., F. Torres, F. Rada \& Y. León. 2007. Estudio morfoanatómico de dos orquídeas de una selva nublada tropical. Interciencia 32: 410-418.

Esau, K. 1965. Anatomy of seed plants. Wiley, Nueva York, EEUU.

Farris, J. 1989. The retention index and rescaled consistency index. Cladistics 5: 417-419.

Goloboff, P. 1993. Nona Ver 1.6. Program and documentation. Distribuido por el autor. Buenos Aires, Argentina.

Haberlandt, G. 1914. Physiological plant anatomy. MacMillan, Londres, Reino Unido.

Jones, H.G. 1975. Nomenclatural revision of the genus Brassavola R. Br. of the Orchidaceae. Ann. Naturhist. Mus. in Wien. 79: 9-22.

Kluge, A.G. \& J.S. Farris. 1969. Quantitative phyletics and evolution of anurans. Syst. Zool. 18: 1-32.
Lindorf, H., L. de Parisca \& P. Rodríguez. 1991. Botánica: clasificación, estructura, reproducción. Ediciones de la Biblioteca, Universidad Central de Venezuela, Caracas D. F., Venezuela.

Madisson, M. 1977. Vascular epiphytes: their systematic occurrence and salient features. Selbyana 2: 1-13.

Nixon, K. 1999. The parsimony Ratchet: A new method for rapid parsimony analysis. Cladistics 15: 207-414.

Nixon, K. 2002. WinClada ver. 1.00.08. Published by the author, Ithaca, Nueva York, EEUU.

Oliveira, V.C. \& M.G. Sajo. 1999. Anatomía foliar de especies epífitas de Orchidaceae. Rev. Bras. Bot. 22: 363-374.

Porembski, S. \& W. Barthlott. 1988. Velamen radicum micromorphology and classification of Orchidaceae. Nord. J. Bot. 8: 117-137.

Pridgeon, A.M. 1986. Anatomical adaptations in Orchidaceae. Lyndleyana 1: 90-101.

Pridgeon, A.M., W.L. Stern \& D. Benzing. 1983. Tilosomes in roots of Orchidaceae: Morphology and systematic occurrence. Am. J. Bot. 70: 1365-1377.

Prychid, C.J., P. Rudall \& M. Gregory. 2004. Systematics and biology of silica bodies in Monocotyledons. Bot. Rew. 69: 377-440.

Pyykkö, M. 1966. The leaf anatomy of east Patagonian xeromorphic plants. Ann. Bot. Fenn. 3: 453-662.

Roth, I. 1992. Leaf structure: coastal vegetation and mangroves of Venezuela. Gebr. Borntraeger, Berlin, Alemania.

Rudall, P. 1992. Anatomy of flowering plants. An introducction to structure and development. Cambridge University, Londres, Reino Unido.

Sandoval-Zapotitla, E., T. Terrazas, G. Salazar, A. Vallejo \& B. Estrada. 2003. Anatomía vegetativa de Mexipedium xerophyticum (Soto, Salazar \& Hágsater) V. A. Albert \& M. W. Chase y géneros relacionados (Orchidaceae, Cypripedioideae). Lankesteriana 7: 54-56.

Sass, J. 1958. Botanical microtechnique. Iowa State College, Iowa, EEUU.

Scatena, V. \& A. Nunes. 1996. Anatomía de Pleurothallis ruprestris Lindl. (Orchidaceae) de dos campos rupestres de Brasil. Bol. Bot. 15: 35-43.

Schlechter, R. 1919. Die Gattung Brassavola R. Br. Orchis 13: 40-77. 
Stern, W.L., W. Judd \& B.S. Carlsward. 2004. Systematic and comparative anatomy of Maxillarieae (Orchidaceae), sans Oncidiinae. Bot. J. Linn. Soc. 144: 251-274.

Stern, W.L. \& B.S. Carlsward. 2006. Comparative vegetative anatomy and systematics of Oncidiinae (Maxillarieae, Orchidaceae). Bot. J. Linn. Soc. 152: 91-107.

Stern, W.L. \& B.S. Carlsward. 2009. Comparative vegetative anatomy and systematic of Laeliinae (Orchidaceae). Bot. J. Linn. Soc. 160: 21-41.

van den Berg, C. 2005. Laeliinae, p. 181-186. In A Pridgeon, P. Cribb, C. Mark \& F. Rammunssen. (eds.). Genera Orchidacearum 4. Epidendroideae (Part. One). Oxford University, Oxford, Reino Unido.

van den Berg, C., W. Higgins, R. Dressler, M. Whitten, M. Soto-Arenas \& M. Chase. 2009. A phylogenetic study of Laeliinae (Orchidaceae) based on combined nuclear and plastid DNA sequences. Ann. Bot. 104: 417-430.

Withner, C., P. Nelson \& P. Wejksnora. 1974. The anatomy of orchids. In the Orchids: scientific studies. Wiley, Nueva York, EEUU.

Withner, C. 1998. The Cattleyas and their relatives, Vol. V. Brassavola, Encyclia and other genera of Mexico and Central America. Timber, Portland, Oregon, EEUU.

Withner, C. 2007. The genus Brassavola revisited. Another look at a confusing taxon. Orchid Digest. 71: 246-255.

Yukawa, T. \& W.L. Stern. 2002. Comparative vegetative anatomy and systematics of Cymbidium (Cymbidieae: Orchidaceae). Bot. J. Linn. Soc. 138: 383-419. 